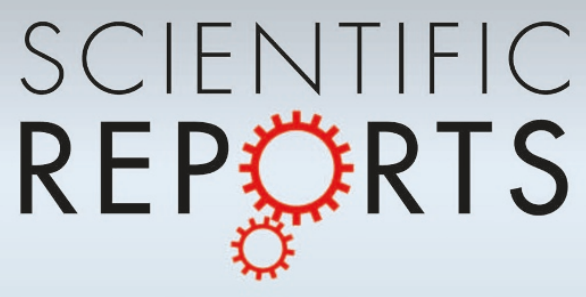

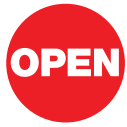

SUBJECT AREAS:

GRANULOCYTES

ANTIGEN-PRESENTING CELLS

FUNGAL HOST RESPONSE

DENDRITIC CELLS

Received

10 December 2012

Accepted

10 January 2013

Published

1 February 2013

Correspondence and requests for materials should be addressed to

J.B. (jagadeesh. bayry@crc.jussieu.fr)

\section{Circulating human basophils lack the features of professional antigen presenting cells}

\author{
Meenu Sharma ${ }^{1,2}$, Pushpa Hegde ${ }^{1,2}$, Vishukumar Aimanianda ${ }^{3}$, Remi Beau ${ }^{3}$, Mohan S. Maddur', \\ Helene Sénéchal ${ }^{4,5}$, Pascal Poncet ${ }^{5,6}$, Jean-Paul Latgé ${ }^{3}$, Srini V. Kaveri ${ }^{1,7,8,9}$ \& Jagadeesh Bayry $1,7,8,9$
}

'Unité 872, Institut National de la Santé et de la Recherche Médicale, Paris, F-75006, France, ${ }^{2}$ Université de Technologie de Compiègne, Compiègne, F-60205, France, ${ }^{3}$ Institut Pasteur, Aspergillus Unit, Paris-75015, France, ${ }^{4}$ Institut National de la Santé et de la Recherche Médicale, Paris-75013, France, ${ }^{5}$ ESPCI ParisTech, UMR 7195 CNRS, Paris-75005, France, ${ }^{6}$ Institut Pasteur, Department of Infection and Epidemiology, Paris-75015 France, ${ }^{7}$ Centre de Recherche des Cordeliers, Equipe 16Immunopathology and therapeutic immunointervention, Université Pierre et Marie Curie - Paris 6, UMR S 872, Paris, F-75006, France, ${ }^{8}$ Université Paris Descartes, UMR S 872, Paris, F-75006, France, ${ }^{9}$ International Associated Laboratory IMPACT (Institut National de la Santé et de la Recherche Médicale, France - Indian council of Medical Research, India), National Institute of Immunohaematology, Mumbai, 400012, India.

Recent reports in mice demonstrate that basophils function as antigen presenting cells (APC). They express MHC class II and co-stimulatory molecules CD80 and CD86, capture and present soluble antigens or IgE-antigen complexes and polarize Th2 responses. Therefore, we explored whether human circulating basophils possess the features of professional APC. We found that unlike dendritic cells (DC) and monocytes, steady-state circulating human basophils did not express HLA-DR and co-stimulatory molecules CD80 and CD86. Basophils remained negative for these molecules following stimulation with soluble Asp $\mathrm{f} 1$, one of the allergens of Aspergillus fumigatus; Bet $\mathrm{v} 1$, the major birch allergen; TLR2-ligand or even upon IgE cross-linking. Unlike DC, Asp f 1-pulsed basophils did not promote Th2 responses as analyzed by the secretion of IL- 4 in the basophil-CD4 ${ }^{+} \mathrm{T}$ cell co-culture. Together, these results demonstrate the inability of circulating human basophils to function as professional APC.

asophils are the rare granulocytes and represent $<1 \%$ of circulating leukocytes. They are Fc\&RI ${ }^{+} \mathrm{CD} 203 \mathrm{c}^{+}$ $\mathrm{CD} 23^{+}$and play an important role in the protection against helminth parasites and in the pathogenesis of allergic diseases and in autoimmune conditions such as lupus nephritis and rheumatoid arthritis ${ }^{1-7}$. Basophils are activated by recognition of protein allergens, proteases and helminth parasite antigens via pattern recognition receptors and FceRI-mediated signaling. The latter is initiated by cross-linking of FceRI either through binding of allergen to surface-bound IgE or by IgE-immune complexes. All these events lead to the secretion of IL-4, IL-13, B cell-activating factor belonging to the TNF family (BAFF) and thymic stromal lymphopoietin (TSLP) that help in the programming of Th2 responses and stimulation of antibody production by $\mathrm{B}$ cell $\mathrm{s}^{3,8-10}$. In addition, basophils also release other inflammatory mediators such as histamine and leukotrines that mediate vasodilation and infiltration of immune cells.

Recent reports have shown that basophils could also act as professional antigen presenting cells (APC) in various murine models ${ }^{8,11-14}$. Professional APC are those that can uptake the antigens, process and present the antigenic peptides in the context of MHC molecules and co-stimulatory molecules to $\mathrm{CD} 4^{+} \mathrm{T}$ cells leading to activation, proliferation and polarization of $\mathrm{CD}^{+} \mathrm{T}$ cell responses ${ }^{15}$. Until now, dendritic cells (DC), macrophages/monocytes and memory B cells were considered as professional APC. However, recent studies in mice have demonstrated that basophils express MHC class II and co-stimulatory molecules CD80 and CD86, capture and present soluble antigens or IgE-antigen complexes and polarize Th2 responses ${ }^{11-13}$. Therefore, in the present report, we explored whether circulating human basophils from healthy individuals possess the features of professional APC and mediate Th2 responses to soluble antigens.

\section{Results}

Circulating human basophils do not express HLA-DR and co-stimulatory molecules CD80 and CD86. We first analyzed the expression of antigen presenting molecule HLA-DR and co-stimulatory molecules of B7 family 
in the steady-state basophils. Previous reports have demonstrated that circulating basophils from naïve mice express detectable levels of these molecules ${ }^{13}$. However, we could not detect the expression of either HLA-DR or co-stimulatory molecules CD80 and CD86 on the circulating basophils (Fig. 1). The expression of these molecules was less than $1 \%$. On the contrary, $100 \%$ of steady-state circulating monocytes and monocyte-derived DC expressed HLA-DR molecules. Although monocytes did not express CD80, nearly $80 \%$ DC expressed this molecule. Further, CD86 was expressed both on monocytes (about 90\%) and on DC (about 30\%) (Fig. 1).

The lack of HLA-DR expression on purified basophils was not due to depletion of these cells during purification process by basophil

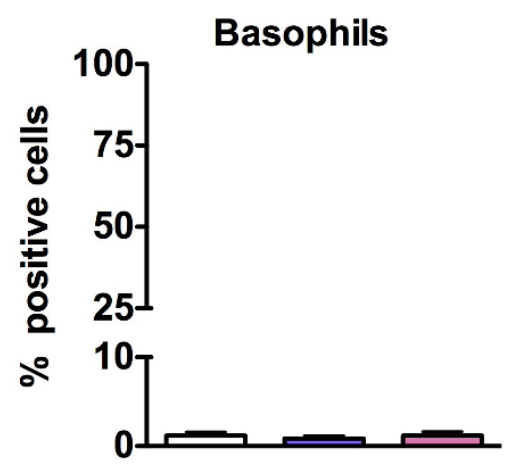

\section{Dendritic cells}
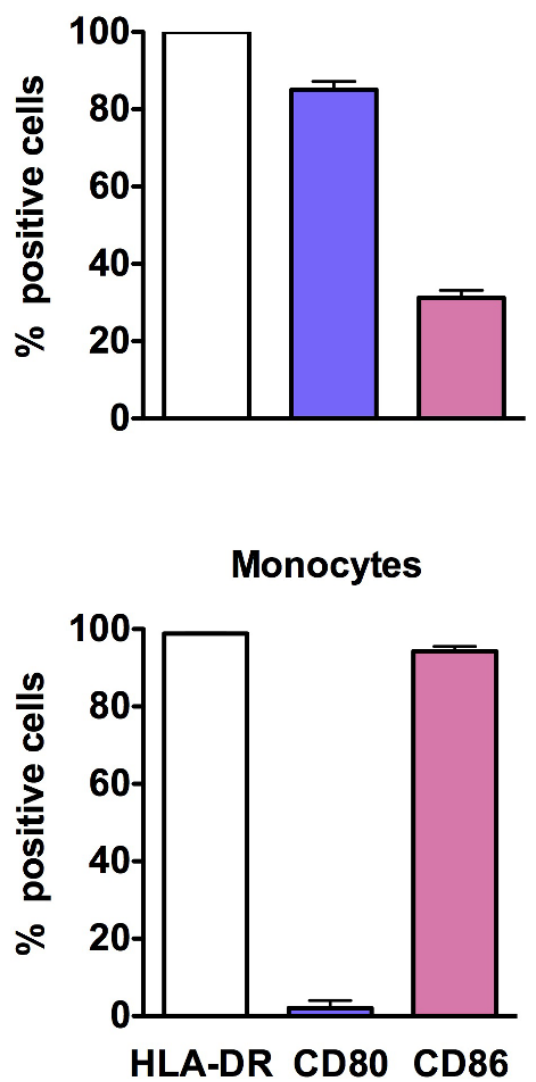

Figure 1 Expression of HLA-DR and co-stimulatory molecules CD80 and CD86 on circulating human basophils, monocytes and monocytederived DC. The expression of markers was determined by flow cytometry by using fluorochrome-conjugated monoclonal antibodies. The results are presented as percentage positive cells. Data (mean \pm SEM) are from three independent donors. isolation kit II (Miltenyi Biotech) that contained anti-HLA-DR antibodies in the antibody cocktail. Analysis of basophils in the blood even before purification revealed a lack of HLA-DR expression (Fig. 2a). In addition, the expression level of HLA-DR was $\leq 1 \%$ on the basophils isolated by EasySep Human Basophil enrichment kit (Stem cell technologies) that did not deplete HLA-DR-expressing basophils (Fig. 2b).

Dendritic cells but not basophils show enhanced expressions of HLA-DR, CD80 and CD86 upon interaction with allergens. When APC are stimulated with antigens, these cells undergo activation and the process is characterized by the enhanced expression of antigenpresenting and co-stimulatory molecules. Therefore we investigated whether interaction of circulating human basophils with allergens leads to induction of HLA-DR, CD80 and CD86 on the cells. We found that stimulation of DC with Asp $\mathrm{f} 1$, one of the major allergens of Aspergillus fumigatus, lead to increased expression of HLA-DR and co-stimulatory molecules (Fig. 3a). On the contrary, basophils remained negative for these molecules (Fig. 3b). As basophils express toll-like receptor 2 (TLR2) ${ }^{16}$, we further examined the expression of HLR-DR, CD80 and CD86 on basophils stimulated with TLR2ligand. However, we did not observe the expression of above molecules on TLR2-stimulated basophils (Fig. 3c and 3d).

We confirmed that basophils were viable and fully functional after stimulation with Asp f 1 . The level of apoptosis in IL-3-treated basophils was in the range of 2.3 to $6.2 \%$ (Fig. 4). Compared to this, apoptosis in Asp f 1-treated basophils was in the range of 1.8 to $4.8 \%$ (Fig. 4). Thus, the level of apoptosis in control and Asp f 1treated basophils did not differ significantly. Further, Asp f 1-treated basophils were functionally viable as determined by the activation status of cells. The levels of expression of activation-associated markers CD63, CD203c and CD13 were similar both in control and in Asp f 1-stimulated basophils upon anti-IgE-stimulation (Fig. 5).

Further, we investigated whether the lack of expression of antigen presenting and co-stimulatory molecules on basophils was restricted to only to Asp $\mathrm{f} 1$ or to other allergens. We analyzed the expression of HLA-DR and co-stimulatory molecules on the basophils stimulated with other well-defined allergens such as Bet $\mathrm{v} 1$, the major birch pollen allergen and Phl p 5, a major timothy grass pollen allergen. The level of apoptosis in Bet $\mathrm{v}$ 1-treated basophils was in a similar range as that of IL-3-treated control basophils (Fig. 4). Also, we did not observe the expression of HLA-DR, CD80 and CD86 on basophils upon stimulation with these allergens (Fig. 6 and data not shown). Similar results were also observed with other antigens of A. fumigatus, superoxide dismutase (Sod1p) and mycelial catalase 1 (Cat1p) (data not shown).

In the context of secondary immune responses, IgE-bound allergens could trigger basophil activation. Therefore, to mimic this situation and to demonstrate unequivocally that the lack of HLA DR, CD80 and CD86 expression on basophils were not restricted to stimulation of cells with a given antigen, we stimulated basophils by IgE crosslinking. However, we could not observe the expression of these molecules on basophils under this stimulation condition (Fig. 6). Together these results indicated that although basophils from mice do express antigen presenting and co-stimulatory molecules, circulating human basophils do not express these molecules under any stimulatory conditions.

Asp f 1-primed dendritic cells but not basophils promote Th2 responses. One of the key functions of APC is to promote polarized $\mathrm{T}$ cell responses. Asp f 1-primed DC or basophils were co-cultured with autologous $\mathrm{CD}^{+} \mathrm{T}$ cells at $1: 40$ ratio and the Th1 and Th2 responses were measured by analyzing the quantities of IFN- $\gamma$ and IL- 4 in the culture supernatants. We show that, DC that were primed with Asp f 1, increased the production of IL-4 from $\mathrm{CD} 4{ }^{+} \mathrm{T}$ cells thus indicated the promotion of Th2 responses by Asp $\mathrm{f}$ 1-primed DC (Fig. 7a). However, Asp f 1-pulsed basophils did not 


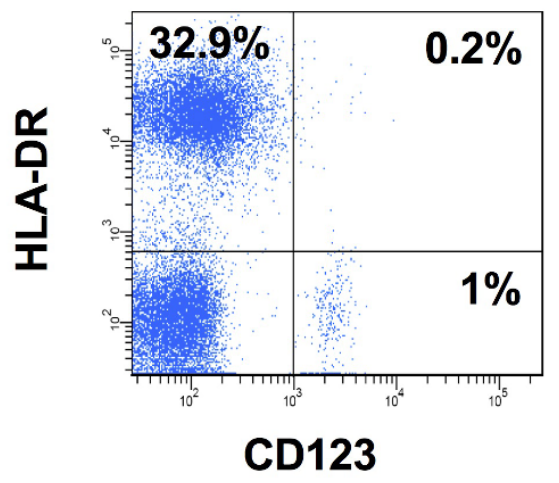

b
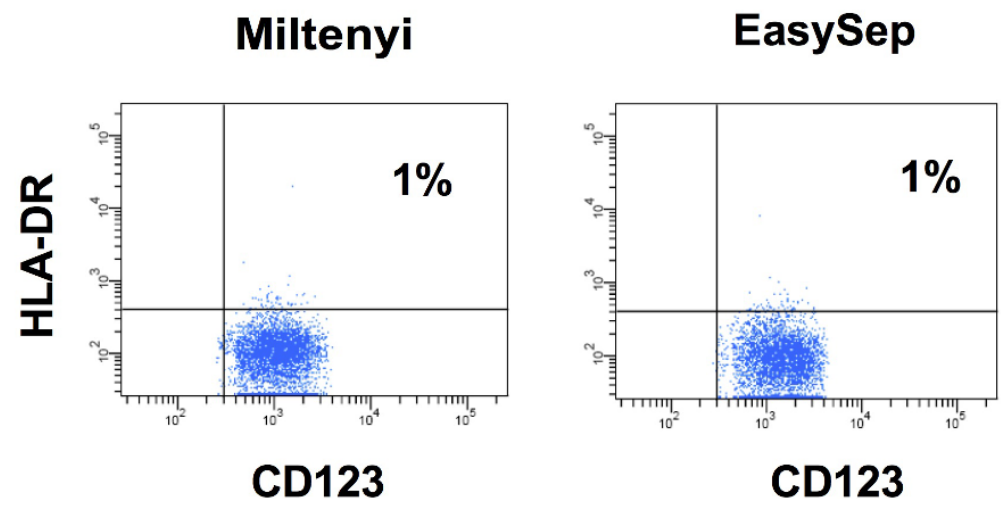

Figure 2 Expression of HLA-DR on basophils in the circulation and in the cells isolated by two different kits. (a) Red blood cells in the buffy bags were lysed and stained for HLA-DR and CD123 (b) Basophils were isolated either by EasySep Human Basophil enrichment kit that does not deplete HLA-DR expressing basophils or by basophil isolation kit II. The expression of HLA-DR and CD123 was analyzed by flow cytometry.

mount Th2 responses. The level of IL- 4 secretion was on par with $\mathrm{CD}^{+} \mathrm{T}$ cells co-cultured with unstimulated basophils or Asp f 1stimulated $\mathrm{CD}^{+}{ }^{+}$T cells (Fig. 7a). The IFN- $\gamma$ production from $\mathrm{CD}^{+}$ $\mathrm{T}$ cells remained unchanged both by DC and basophils (Fig. 7b). Together, these results confirmed the inability of circulating human basophils to function as professional APC.

\section{Discussion}

The main function of professional APC is to present the antigen to $\mathrm{CD}^{+} \mathrm{T}$ cells and to polarize $\mathrm{T}$ cell responses at low APC $: \mathrm{CD} 4^{+} \mathrm{T}$ cell ratios ${ }^{15}$. APC-mediated $\mathrm{CD} 4^{+} \mathrm{T}$ cell polarization implicates four different signals: signal zero at the interface of APC and the antigen, signal 1 at the interface of antigen-loaded HLA-DR on APC with T cell receptor-CD3 complexes on T cells, signal 2 that implicates the interaction of co-stimulatory molecules CD80/CD86 on APC with $\mathrm{CD} 28$ of $\mathrm{T}$ cells and signal 3 in the form of $\mathrm{CD}^{+} \mathrm{T}$ cell-polarizing cytokines secreted by APC. Although, human basophils secrete high amounts of Th2-polarizing cytokine IL-4, our results demonstrate that unlike other professional APC, circulating human basophils lack signal 1 and signal 2 that are implicated in T cell polarization.

Previous studies by using basophils from allergic patients have demonstrated that human basophils do not internalize and present IgE-bound antigens ${ }^{17,18}$. These reports however studied the function of basophils in the context of secondary immune responses and did not confirm whether human circulating basophils could present soluble antigens in the context of primary immune responses to polarize $\mathrm{T}$ cell responses. In addition, whether direct stimulation of circulating human basophils with an allergen could lead to expression of antigen presenting and co-stimulatory molecules was also not addressed. Professional APC could internalize not only immune complexes but also soluble antigens via receptor-mediated endocytosis, pinocytosis and phagocytosis ${ }^{19}$. The signaling via antigens (such as pathogen-associated molecular patterns) leads to activation of APC with enhanced expression of antigen-presenting molecules and co-stimulatory molecules. Basophils did not express these molecules despite priming with Asp f 1, Bet v 1 and other allergens. As a consequence, unlike DC, basophils did not polarize Th2 responses (as measured by IL-4). Even at higher ratios of basophils : $\mathrm{CD}^{+} \mathrm{T}$ cells $(1: 10)$ there were no apparent polarization of Th2 responses. On the other hand, DC were activated by Asp f 1 and induced Th2 polarization. These results thus confirmed the previous reports that DC could mediate allergic immune responses upon interaction with allergens $^{20}$.

A. fumigatus is one of the major fungal species implicated in the pathogenesis of several respiratory diseases including asthma and allergic broncho-pulmonary aspergillosis (ABPA) $)^{21}$. Asp $\mathrm{f} 1$ is a $18 \mathrm{kd}$ ribonuclease protein of ribotoxin family and is one of the major allergens of Aspergillus. Presence of IgE-reacting to Asp $f 1$ is a 
a
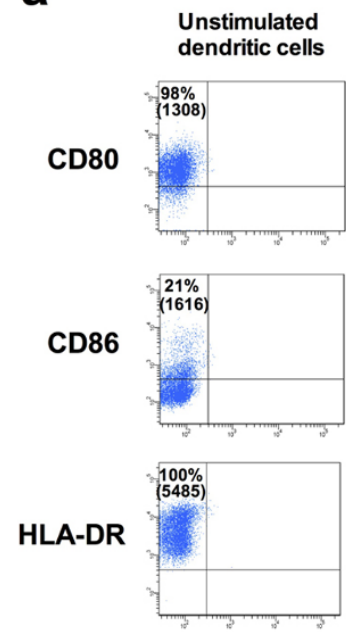

APC

C
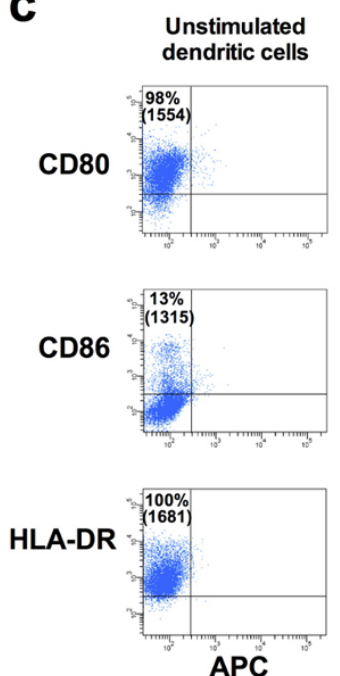
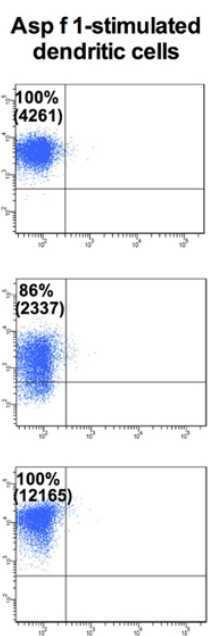

APC
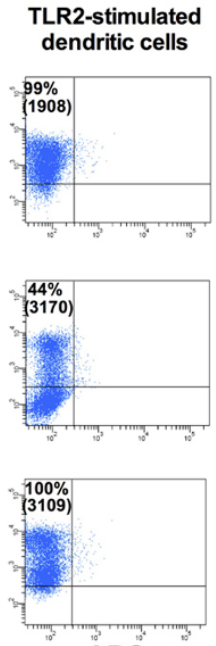

APC b
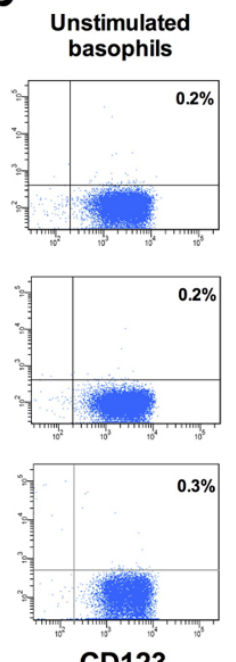

CD123

d
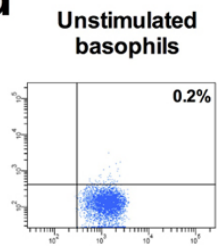

TLR2-stimulated basophils
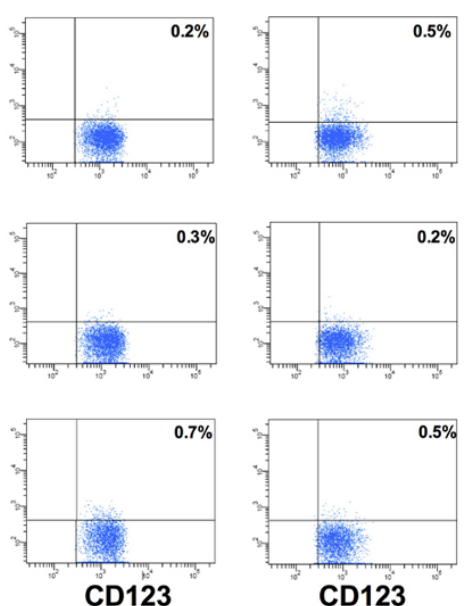

Figure 3 Monocyte-derived DC but not basophils show enhanced expressions of HLA-DR, CD80 and CD86 upon stimulation with Asp f 1 and peptidoglycan. (a and c) Monocyte-derived DC were cultured in X-Vivo medium containing 5\% human AB serum and cytokines GM-CSF and IL-4 (panel: unstimulated dendritic cells) or cytokines plus Asp f 1 (panel: Asp f 1-stimulated dendritic cells) or cytokines plus peptidoglycan (panel: TLR2stimulated dendritic cells) for 24 hours. The phenotype of DC was analyzed by flow cytometry. (b and d) Basophils were cultured in X-Vivo medium containing 5\% human AB serum and IL-3 alone (panel: unstimulated basophils) or IL-3 plus Asp f 1 (panel: Asp f 1-stimulated basophils) or IL-3 plus peptidoglycan (panel: TLR2-stimulated basophils) for 24 hours. The phenotype of basophils was analyzed by flow cytometry. Percentage of cells expressing indicated markers and mean fluorescence intensity values (in parenthesis) are indicated. Results are representative of at least three experiments.

common feature of ABPA and allergic asthma ${ }^{22,23}$. Previous studies have also demonstrated that Asp $\mathrm{f} 1$ is highly immunogenic and could activate APC such as DC to induce maturation of cells as well as proinflammatory cytokine and chemokine responses ${ }^{24,25}$. However, in the air-borne dormant conidia, Asp f 1 is covered by hydrophobin layer that masks Asp f 1 being recognized by innate cells ${ }^{24,26}$.

However, our present results demonstrate that Asp $\mathrm{f} 1$ could not be presented by circulating human basophils to initiate immune responses. As Asp f 1-pulsed DC promoted Th2 responses, these results indicate that IgE antibody responses to Asp $\mathrm{f} 1$ in ABPA and allergic asthma patients could be mediated mainly via DC. Also data from experimental models (DC vaccination model) have shown that Asp $\mathrm{f}$ 1 induces Th2 responses ${ }^{27}$.

Protease allergens could trigger innate responses including DC via protease-activated receptors (PARs) $)^{28-30}$ and fungal proteases were proposed to initiate inflammatory responses via $\mathrm{PARs}^{31-33}$. Therefore, based on these data, it is likely that Asp $\mathrm{f} 1$ could activate DC via PAR signaling ${ }^{34}$. However, PARs were found to be absent on human basophils ${ }^{35}$ and hence potential innate trigger for protease allergens on basophils is still a mystery. However, in the context of secondary immune responses, IgE-bound allergens could trigger basophil activation. But, we did not observe the expression of antigen presenting and co-stimulatory molecules on basophils even upon IgE crosslinking. Similarly, TLR2 stimulation did not induce expression of HLA-DR, CD86 and CD80 on basophils.

We used percoll density gradient of three different densities $(1.070,1.079,1.088)$ for obtaining basophil rich peripheral blood mononuclear cells (PBMC) ${ }^{36}$ and bead-based negative isolation method to isolate circulating human basophils from PBMC. Basophils in the circulation were in the range of 0.4 to $1 \%$ and in 


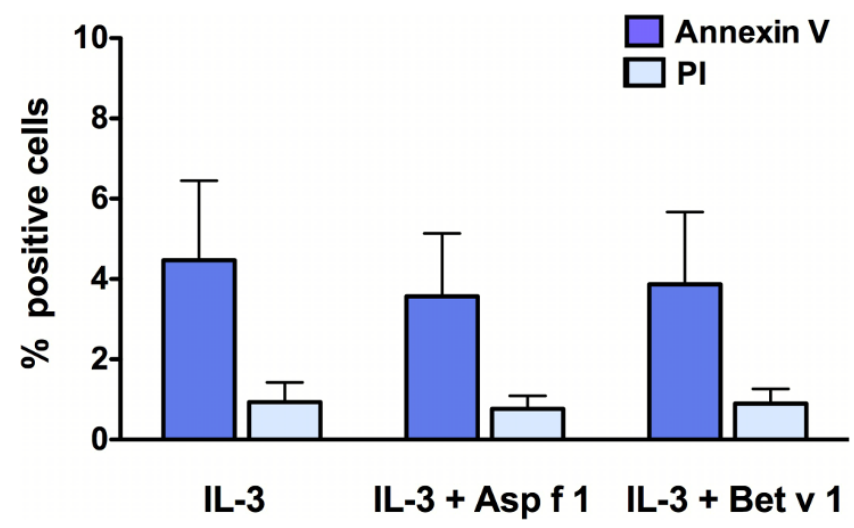

Figure $4 \mid$ The viability of basophils. Basophils were cultured in complete medium containing IL-3 alone or with Asp f 1 or Bet $\mathrm{v} 1$ for 24 hours. Viability of basophils was analyzed by surface expression of apoptotic maker, Annexin V and necrotic marker, propidium iodide (PI). Data (mean \pm SEM) are from three independent experiments.

the percoll purified PBMC, they were in the range of 0.5 to $1.2 \%$. The recovery of basophils by using negative selection on a MACS system was in the range of $0.3-1.5 \%$. However, the yield was lower with EasySep Human Basophil enrichment kit (0.05-0.2\%). The purity of isolated basophil population was generally $>95 \%$ as analyzed by combination of various markers such as CD203c and CD123. There were no contaminating $\mathrm{CD} 14^{+}$cells in the isolated populations. Also, the lack of polarization of $\mathrm{CD}^{+}{ }^{+} \mathrm{T}$ cells in the co-culture of $\mathrm{CD} 4^{+} \mathrm{T}$ cells with Asp f 1-primed basophils, indirectly ruled out the presence of any contaminating APC in the basophil population.

Most of the results in murine models come from basophils isolated from either lymph nodes or spleen following antigen stimulation ${ }^{11-13}$. Although basophils are circulating cells and normally not tissue resident, it is not known whether migration of human basophils to lymphoid tissues could alter the phenotype and expression of antigen presenting and co-stimulatory molecules. Therefore, further studies on lymphoid tissue basophils are required to support our observations.

\section{Methods}

Isolation of circulating human basophils, monocytes and generation of monocyte-derived DC. Buffy bags from the healthy donors were obtained from Hôpital Hôtel Dieu, Etablissement Français du Sang, Paris, France. Ethical committee permission was obtained for the use of buffy bags of healthy donors. Basophil-rich fraction of PBMC was obtained by percoll density gradient centrifugation ${ }^{36}$. Basophils from these basophil-rich PBMC were isolated either by basophil isolation kit II (Miltenyi Biotech, Paris, France) or EasySep human basophils enrichment kit (Stem cell technologies, Grenoble, France). Monocytes were isolated from PBMC by using CD14 magnetic micro-beads (Miltenyi Biotec). The monocytes were cultured in GMCSF $\left(1000 \mathrm{IU} / 10^{6}\right.$ cells) and IL-4 (500 IU $/ 10^{6}$ cells) (both from Miltenyi Biotec) for five to six days to generate $\mathrm{DC}^{37}$.

Stimulation of cells. Immature DC were stimulated either with recombinant Asp f 1 $\left(1 \mu \mathrm{g} / 0.5 \times 10^{6}\right.$ cells $)$ or with peptidoglycan $\left(5 \mu \mathrm{g} / 0.5 \times 10^{6}\right.$ cells $)$ in the X-Vivo medium containing $5 \% \mathrm{AB}$ serum, IL-4 and GM-CSF. The recombinant Asp f 1 protein (18-kDa RNase, Afu 5g02330) from A. fumigatus was produced as previously described $^{38}$. The purity of protein was analyzed by SDS-PAGE followed by revealing the protein band by silver staining and MALDI-TOF mass spectrometry. After 24 hours stimulation, DC were washed two times with large amounts of RPMI 1640 to remove residual IL-4 and GM-CSF. The cells were then processed either for flow cytometry or co-culture with $\mathrm{CD}^{+} \mathrm{T}$ cells.

Basophils were cultured in X-Vivo medium containing 5\% human AB serum. IL-3 $\left(100 \mathrm{ng} / 10^{6}\right.$ cells $)$ was also added to the medium in order to maintain viability of the cells. The cells were stimulated for 24 hours with peptidoglycan $(5 \mu \mathrm{g} / 0.5 \times$ $10^{6}$ cells), recombinant Asp f 1 , Sod1p, Cat1p, Bet v 1, Phl p 5 (all at $1 \mu \mathrm{g} / 0.5 \times$ $10^{6}$ cells). Recombinant Sod1p (Afu 5g09240) and Catlp (Afu 3g02270) from A. fumigatus were produced as previously described ${ }^{38}$ and recombinant Bet $\mathrm{v} 1$ and Phl 55 were kind gift from ALK-Abello (Copenhagen, Denmark). The cells were then used either for flow cytomtery or co-culture with $\mathrm{CD} 4^{+} \mathrm{T}$ cells.

The viability of allergen-pulsed cells was analyzed by surface expression of apoptotic maker, Annexin V and necrotic marker, propidium iodide (PI). To determine
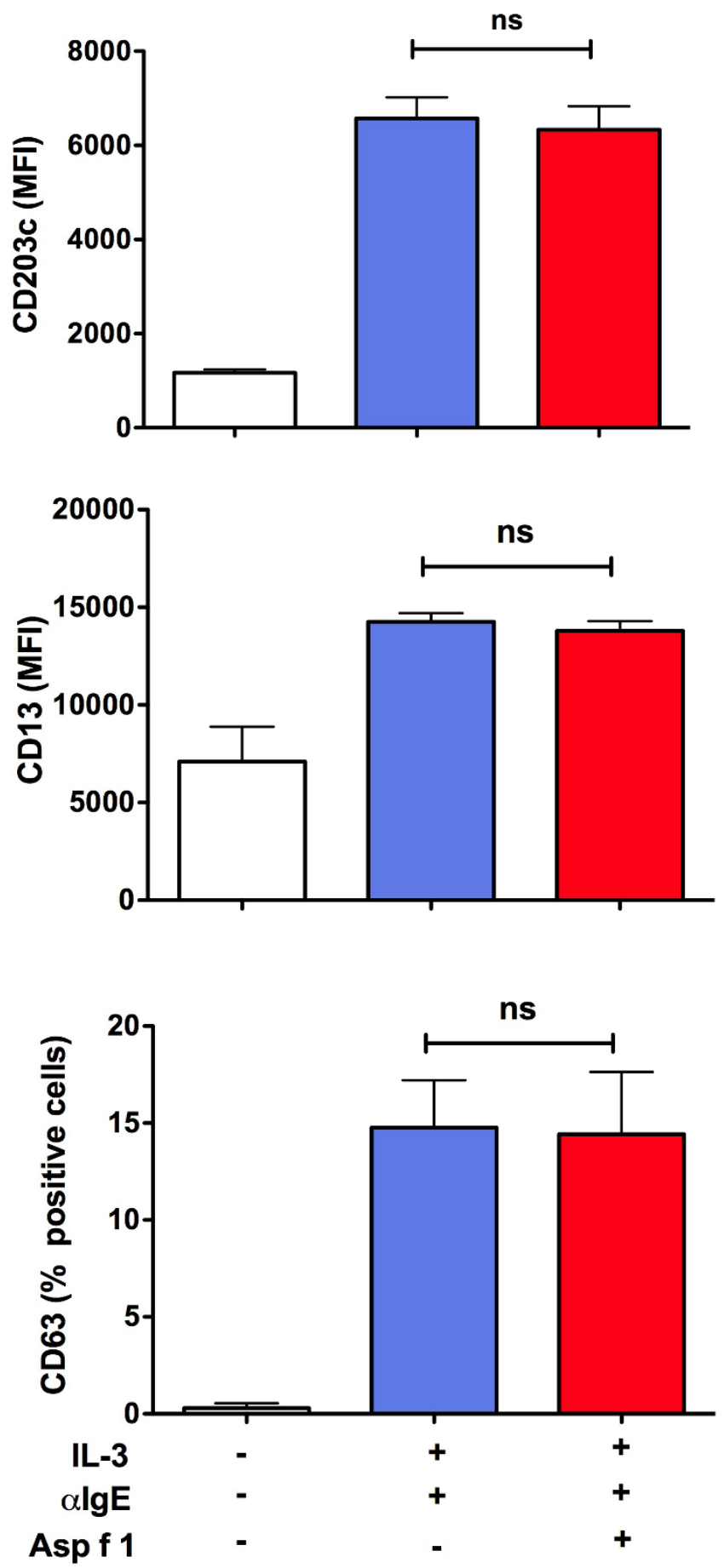

Figure 5 $\mid$ Asp f 1-treated basophils are functionally viable. Basophils were cultured with or without Asp f 1 in the presence of IL-3 for 24 hours. These cells were stimulated with anti-IgE antibodies (10 ng/ml) during last one hour of the culture. Basophil response was determined by analyzing the expression of activation-associated markers CD203c, CD13 and CD63 by flow cytometry. Data (mean \pm SEM) are from three independent experiments.

whether Asp f 1-treated basophils were functionally viable, basophils were cultured with or without Asp $\mathrm{f} 1$ in the presence of IL-3 for 24 hours. These cells were stimulated with anti-IgE antibodies $(10 \mathrm{ng} / \mathrm{ml})$ during last one hour of the culture. Basophil response was determined by analyzing the expression of activation-associated markers CD63, CD203c and CD13 by flow cytometry.

Co-culture of basophils or DC with $\mathrm{CD}^{+} \mathrm{T}$ cells. Autologous $\mathrm{CD} 4^{+} \mathrm{T}$ cells were negatively isolated from PBMC by using $\mathrm{CD}^{+} \mathrm{T}$ cell isolation kit II from Miltenyi Biotech. The $\mathrm{CD}^{+} \mathrm{T}$ cells $\left(0.1 \times 10^{6}\right.$ cells/well in U-bottomed 96 wells plate $)$ were 


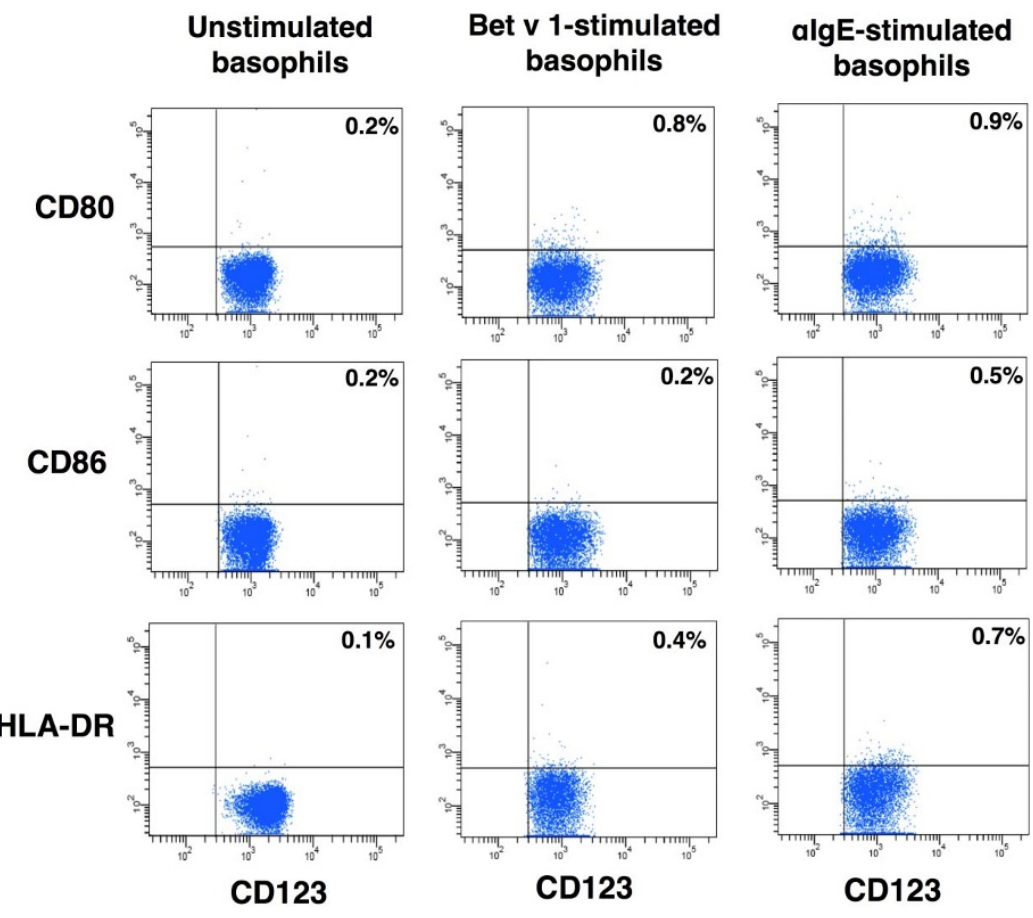

Figure 6 The lack of expression of antigen presenting and co-stimulatory molecules on basophils is a universal phenomenon. Basophils were cultured in X-Vivo medium containing 5\% human AB serum and IL-3 alone (panel: unstimulated basophils) or IL-3 plus Bet v 1 or IL-3 plus anti-IgE antibodies for 24 hours. The phenotype of basophils was analyzed by flow cytometry. Percentage of cells expressing indicated markers are indicated. Results are representative of three experiments.

a

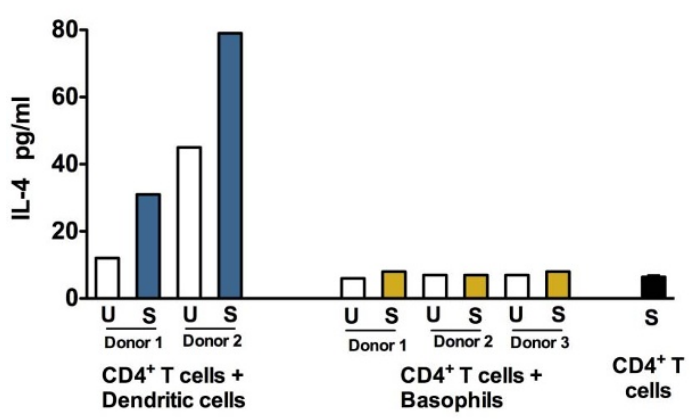

b

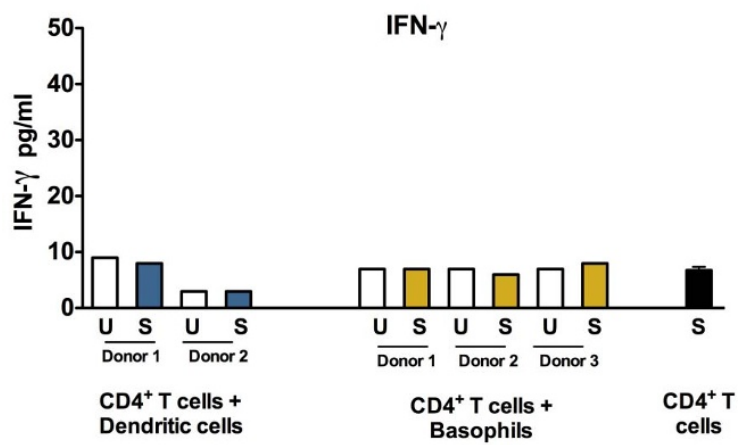

Figure $7 \mid$ Asp f 1-primed monocyte-derived dendritic cells but not basophils promote $\mathrm{Th} 2$ responses. The autologous $\mathrm{CD} 4^{+} \mathrm{T}$ cells were co-cultured with Asp f 1-pulsed (labeled as S) or unpulsed (labeled as U) basophils (Panel: $\mathrm{CD}^{+}{ }^{+}$T cells + Basophils) or monocyte-derived DC (Panel: $\mathrm{CD}^{+} \mathrm{T}$ cells + Dendritic cells) at $40: 1$ ratio for 4 days in X-Vivo medium containing $5 \%$ human $\mathrm{AB}$ serum. The amount of IL-4 (a) and IFN- $\gamma(\mathrm{b})$ in the cell-free culture supernatants was measured. co-cultured with Asp f 1-pulsed or unpulsed basophils or DC at $40: 1$ ratio for 4 days in X-Vivo medium containing 5\% human AB serum. The cell-free culture supernatants were used for measuring the T cell cytokines. Asp f 1-pulsed or unpulsed $\mathrm{CD} 4^{+} \mathrm{T}$ cells (without DC or basophils) were used as controls.

Flow cytometry. The cell surface staining was performed by using fluorochromeconjugated monoclonal antibodies to CD80, CD86, HLA-DR, CD14, CD63, CD13, Annexin V (all from BD Biosciences, Le Pont de Claix, France), CD203c (Beckman Coulter, Villepinte, France) and CD123 (Miltenyi Biotech). PI was purchased from Sigma-Aldrich (Lyon, France). The cells were then processed for flow cytometry (LSR II, BD Biosciences) and data were analyzed by BD FACS DIVA program.

Cytokine analysis. IFN- $\gamma$ and IL- 4 were measured in cell-free culture supernatants by using $\mathrm{BD}^{\mathrm{TM}}$ cytometric bead array human Th1/Th2 cytokine kit. The detection limits of IFN- $\gamma$ and IL- 4 were $7 \mathrm{pg} / \mathrm{ml}$ and $2.6 \mathrm{pg} / \mathrm{ml}$ respectively.

1. Mukai, K. et al. Basophils play a critical role in the development of IgE-mediated chronic allergic inflammation independently of $\mathrm{T}$ cells and mast cells. Immunity 23, 191-202 (2005).

2. Ohnmacht, C. \& Voehringer, D. Basophil effector function and homeostasis during helminth infection. Blood 113, 2816-2825 (2009).

3. Karasuyama, H., Mukai, K., Obata, K., Tsujimura, Y. \& Wada, T. Nonredundant roles of basophils in immunity. Annu. Rev. Immunol. 29, 45-69 (2011).

4. Charles, N., Hardwick, D., Daugas, E., Illei, G. G. \& Rivera, J. Basophils and the T helper 2 environment can promote the development of lupus nephritis. Nat. Med. 16, 701-707 (2010)

5. Kaveri, S. V., Mouthon, L. \& Bayry, J. Basophils and nephritis in lupus. N. Engl. J. Med. 363, 1080-1082 (2010).

6. Bosch, X., Lozano, F., Cervera, R., Ramos-Casals, M. \& Min, B. Basophils, IgE, and autoantibody-mediated kidney disease. J. Immunol. 186, 6083-6090 (2011).

7. Schuerwegh, A. J. et al. Evidence for a functional role of IgE anticitrullinated protein antibodies in rheumatoid arthritis. Proc. Natl. Acad. Sci. US A 107 (2010).

8. Sokol, C. L. \& Medzhitov, R. Emerging functions of basophils in protective and allergic immune responses. Mucosal Immunol. 3, 129-137 (2010).

9. Denzel, A. et al. Basophils enhance immunological memory responses. Nat. Immunol. 9, 733-742 (2008).

10. van Panhuys, N. et al. Basophils are the major producers of IL-4 during primary helminth infection. J. Immunol. 186, 2719-2728 (2011).

11. Perrigoue, J. G. et al. MHC class II-dependent basophil-CD4+ T cell interactions promote $\mathrm{T}(\mathrm{H}) 2$ cytokine-dependent immunity. Nat. Immunol. 10, $697-705$ (2009)

12. Sokol, C. L. et al. Basophils function as antigen-presenting cells for an allergen-induced T helper type 2 response. Nat. Immunol. 10, 713-720 (2009). 
13. Yoshimoto, T. et al. Basophils contribute to T(H)2-IgE responses in vivo via IL-4 production and presentation of peptide-MHC class II complexes to CD4+ T cells. Nat. Immunol. 10, 706-712 (2009).

14. Maddur, M. S., Kaveri, S. V. \& Bayry, J. Basophils as antigen presenting cells. Trends Immunol. 31, 45-48 (2010).

15. Banchereau, J. \& Steinman, R. M. Dendritic cells and the control of immunity. Nature 392, 245-252 (1998).

16. Bieneman, A. P., Chichester, K. L., Chen, Y. H. \& Schroeder, J. T. Toll-like receptor 2 ligands activate human basophils for both IgE-dependent and IgE-independent secretion. J Allergy Clin Immunol 115, 295-301 (2005).

17. Kitzmuller, C. et al. Human blood basophils do not act as antigen-presenting cells for the major birch pollen allergen Bet v 1. Allergy 67, 593-600 (2012).

18. Eckl-Dorna, J. et al. Basophils are not the key antigen-presenting cells in allergic patients. Allergy 67, 601-608 (2012).

19. Trombetta, E. S. \& Mellman, I. Cell biology of antigen processing in vitro and in vivo. Annu. Rev. Immunol. 23, 975-1028 (2005).

20. Gill, M. A. The role of dendritic cells in asthma. J. Allergy Clin. Immunol. 129, 889-901 (2012).

21. Latge, J. P. Aspergillus fumigatus and aspergillosis. Clin. Microbiol. Rev. 12, 310-350 (1999).

22. Kurup, V. P. et al. Detection of immunoglobulin antibodies in the sera of patients using purified latex allergens. Clin. Exp. Allergy 30, 359-369 (2000).

23. Kurup, V. P. Aspergillus antigens: which are important? Med. Mycol. 43 Suppl 1, S189-196 (2005).

24. Aimanianda, V. et al. Surface hydrophobin prevents immune recognition of airborne fungal spores. Nature 460, 1117-1121 (2009).

25 . Ok, M. et al. Immune responses of human immature dendritic cells can be modulated by the recombinant Aspergillus fumigatus antigen Aspf1. Clin. Vaccine Immunol. 16, 1485-1492 (2009).

26. Bayry, J., Aimanianda, V., Guijarro, J. I., Sunde, M. \& Latge,J. P. Hydrophobins-unique fungal proteins. PLoS Pathog 8, e1002700 (2012).

27. Bozza, S. et al. Immune sensing of Aspergillus fumigatus proteins, glycolipids, and polysaccharides and the impact on Th immunity and vaccination. J Immunol 183, 2407-2414 (2009).

28. Kauffman, H. F. \& van der Heide, S. Exposure, sensitization, and mechanisms of fungus-induced asthma. Curr Allergy Asthma Rep 3, 430-437 (2003).

29. Coughlin, S. R. Thrombin signalling and protease-activated receptors. Nature 407, 258-264 (2000).

30. Ossovskaya, V. S. \& Bunnett, N. W. Protease-activated receptors: contribution to physiology and disease. Physiol Rev 84, 579-621 (2004).
31. Kauffman, H. F., Tomee, J. F., van de Riet, M. A., Timmerman, A. J. \& Borger, P. Protease-dependent activation of epithelial cells by fungal allergens leads to morphologic changes and cytokine production. J Allergy Clin Immunol 105 , $1185-1193$ (2000).

32. Reed, C. E. \& Kita, H. The role of protease activation of inflammation in allergic respiratory diseases. J Allergy Clin Immunol 114, 997-1008 (2004).

33. Moretti, S. et al. The contribution of PARs to inflammation and immunity to fungi. Mucosal Immunol 1, 156-168 (2008).

34. Lewkowich, I. P. et al. Protease-activated receptor 2 activation of myeloid dendritic cells regulates allergic airway inflammation. Respir Res 12, 122 (2011)

35. Falcone, F. H., Morroll, S. \& Gibbs, B. F. Lack of protease activated receptor (PAR) expression in purified human basophils. Inflamm Res 54 Suppl 1, S13-14 (2005).

36. Metcalfe, D. D. Isolation of human basophils. Curr. Protoc. Immunol. Chapter 7 , Unit 724 (2001).

37. Maddur, M. S., Kaveri, S. V. \& Bayry, J. Regulation of human dendritic cells by B cells depends on the signals they receive. Blood 119, 3863-3864 (2012).

38. Sarfati, J. et al. Recombinant antigens as diagnostic markers for aspergillosis. Diagn. Microbiol. Infect. Dis. 55, 279-291 (2006).

\section{Acknowledgements}

Supported by European Community's Seventh Framework Programme [FP7/2007-2013] under Grant Agreement No: 260338 ALLFUN and ANR-10-BLAN-1309 HYDROPHOBIN

\section{Author contributions}

.B. designed research, M.S. \& P.H. performed research, all authors analyzed the data, V.A., R.B., H.S., P.P. \& J.-P.L. provided new research tools. J.B. wrote the paper and all authors reviewed and approved the manuscript.

\section{Additional information}

Competing financial interests: The authors declare no competing financial interests.

License: This work is licensed under a Creative Commons

Attribution-NonCommercial-NoDerivs 3.0 Unported License. To view a copy of this license, visit http://creativecommons.org/licenses/by-nc-nd/3.0/

How to cite this article: Sharma, M. et al. Circulating human basophils lack the features of professional antigen presenting cells. Sci. Rep. 3, 1188; DOI:10.1038/srep01188 (2013) 
SUBJECT AREAS: ANTIGEN-PRESENTING

CELLS

FUNGAL HOST RESPONSE

GRANULOCYTES

DENDRITIC CELLS

\section{SCIENTIFIC REPORTS:}

$3: 1188$

DOI: $10.1038 /$ srep0 1188

(2013)

Published:

1 February 2013

Updated:

15 August 2013

\section{CORRIGENDUM: Circulating human basophils lack the features of professional antigen presenting cells}

\author{
Meenu Sharma 1,2, Pushpa Hegde 1,2, Vishukumar Aimanianda 3 , Remi Beau ${ }^{3}$, Mohan S. Maddur', \\ Helene Sénéchal ${ }^{4,5}$, Pascal Poncet ${ }^{5,6}$, Jean-Paul Latgé ${ }^{3}$, Srini V. Kaveri ${ }^{1,7,8,9}$ \& Jagadeesh Bayry ${ }^{1,7,8,9}$
}

\begin{abstract}
'Unité 872, Institut National de la Santé et de la Recherche Médicale, Paris, F-75006, France, ${ }^{2}$ Université de Technologie de Compiègne, Compiègne, F-60205, France, ${ }^{3}$ Institut Pasteur, Aspergillus Unit, Paris-75015, France, ${ }^{4}$ Institut National de la Santé et de la Recherche Médicale, Paris-75013, France, ${ }^{5}$ ESPCI ParisTech, UMR 7195 CNRS, Paris-75005, France, ${ }^{6}$ Institut Pasteur, Department of Infection and Epidemiology, Paris-75015 France, ${ }^{7}$ Centre de Recherche des Cordeliers, Equipe 16Immunopathology and therapeutic immunointervention, Université Pierre et Marie Curie-Paris 6, UMR S 872, Paris, F-75006, France, ${ }^{8}$ Université Paris Descartes, UMR S 872, Paris, F-75006, France, ${ }^{9}$ International Associated Laboratory IMPACT (Institut National de la Santé et de la Recherche Médicale, France - Indian council of Medical Research, India), National Institute of Immunohaematology, Mumbai, 400012, India.
\end{abstract}

Mohan S Maddur was omitted from the author list in the original version of this Article. This has been corrected in the PDF and HTML versions of the Article.

The Author Contributions section should read "J.B. designed research, M.S., P.H. \& M.S.M. performed research, all authors analysed the data, V.A., R.B., H.S., P.P. \& J.-P.L. provided new research tools. J.B. wrote the paper and all authors reviewed and approved the manuscript." 